\title{
Operational Strategies for Predictive Dispatch of Control Reserves in View of Stochastic Generation
}

Delikaraoglou, Stefanos; Heussen, Kai; Pinson, Pierre

Published in:

Proceedings of 18th Power Systems Computation Conference (PSCC'14)

Publication date:

2014

Link back to DTU Orbit

Citation $(A P A)$ :

Delikaraoglou, S., Heussen, K., \& Pinson, P. (2014). Operational Strategies for Predictive Dispatch of Control Reserves in View of Stochastic Generation. In Proceedings of 18th Power Systems Computation Conference (PSCC'14) IEEE.

\section{General rights}

Copyright and moral rights for the publications made accessible in the public portal are retained by the authors and/or other copyright owners and it is a condition of accessing publications that users recognise and abide by the legal requirements associated with these rights.

- Users may download and print one copy of any publication from the public portal for the purpose of private study or research.

- You may not further distribute the material or use it for any profit-making activity or commercial gain

- You may freely distribute the URL identifying the publication in the public portal

If you believe that this document breaches copyright please contact us providing details, and we will remove access to the work immediately and investigate your claim. 


\title{
Operational Strategies for Predictive Dispatch of Control Reserves in View of Stochastic Generation
}

\author{
Stefanos Delikaraoglou, Kai Heussen and Pierre Pinson \\ Technical University of Denmark \\ Kgs. Lyngby, Denmark \\ Email: $\{$ stde $\},\{$ kh $\},\{$ ppin $\} @$ elektro.dtu.dk
}

\begin{abstract}
In view of the predictability and stochasticity of wind power generation, transmission system operators (TSOs) can benefit from predictive dispatch of slow and manual control reserves in order to maintain reactive reserve levels for unpredictable events. While scenario-based approaches for stochastic optimization are well suited for this problem, it appears that TSOs are hesitant in adopting this method into their practice of predictive dispatch. Differences in the formulation of constraints and cost functions, the timing and reserve product constraints influence the dispatch result significantly and yield varying results with different practical implications. To support adoption, there is a need to study relevant parameters and trade-offs to be considered in introducing such methods to operation practice, enabling also the investigation of alternate reserve product constraints, e.g., to enable reserve contribution from storageconstrained units.

This paper introduces a framework for comparison of operational strategies for system balancing, proposes criteria for performance assessment and exemplifies a systematic evaluation of several operation strategies.
\end{abstract}

\section{INTRODUCTION}

The growth of renewable energy sources (RES) in recent years has been important throughout Europe. The power generation from many RES, e.g., wind power, is largely variable and only partly predictable. This uncertainty poses new challenges on scheduling and dispatch and it increases the need for balancing power.

The two main trading floors of the European electricity markets are the day-ahead market, cleared 12 to 36 hours before the actual operation, and the intra-day market, cleared one hour before operation, which leave portfolio balancing to the market parties. Transmission System Operators (TSOs) have to maintain the continuous system balance by activating manual, i.e., RR, and automatic reserves, i.e., FCR and FRR (see Section II-A), which are procured in ancillary service markets [1].

This market design is suitable for electricity generation based on conventional power plants, where operation schedules need to be coordinated and planned ahead and reserve needs are driven by the outages of generation units or transmission lines. In this conventional operation paradigm, system balancing is mainly reactive and restorative, i.e., manual reserves are dispatched upon observed imbalances to restore

Paper submitted to Power Systems Computation Conference, August 18 22, 2014, Wroclaw, Poland, organized by Power Systems Computation Conference and Wroclaw University of Technology. the availability of automatic reserves. In power systems with high shares of intermittent RES, generation becomes strongly fluctuating and only partly predictable, so that the conditions for the conventional operation paradigm are no longer satisfied. Consequently, modified operational strategies are needed to adapt to increased fluctuation and partial predictability.

Uncertainty in dispatch problems has been considered in the context of scheduling and dynamic reserve requirements, including [2]-[6]. References [2]-[4] formulate a stochastic programming model for joint optimization of day-ahead and balancing operation minimizing the expected cost of the system, while [5] employs adaptive robust optimization in order to minimize the cost of the worst case scenario. Using wind power probabilistic forecasts, [6] defines multi-criteria decision strategies for setting reserve requirements considering risk and economic aspects.

The activation of control reserves can be altered from reactive and restorative to predictive dispatch where control resources are allocated proactively using forecasts with smaller prediction errors. Facing a wind power penetration above $20 \%$, the Danish TSO, Energinet.dk, employs predictive dispatch of regulating power using RES forecasts. By placing emphasis on manual reserves the use of automatic reserves is reduced. This provides: i) reliability benefits since automatic reserves remain available for unpredictable contingencies and ii) cost reduction due to the deployment of cheaper manual reserves [7]. To assess the reserve demands in future scenarios, the "SimBa" model [7] computes this balancing power utilization and dispatch using a rule-based dispatch model.

Improving the availability of balancing resources is expected to reduce scarcity and therefore decrease the balancing costs [8]. To that effect, product constraints such as ramp rates, minimum dispatch volume or activation time may be modified to enable contributions from new flexible demand or generation. For instance, the "Flexiramp" market design [9], [10] adopted by CalISO aims to cover steep ramps and large deviations of wind power; in the PJM electricity market, new products have been designed in order to enable balancing services from demand response (DR) and electric vehicles (EVs) [11]. Cross-border exchange of balancing power is practiced in Europe in the Nordic region for manual reserves via the harmonized and integrated regulating power market. Cooperation on balancing reserves and harmonization also requires revision of the balancing control objectives as well 
as revised market coupling mechanisms, which are beyond the scope of this paper.

This work, as [7], considers predictive dispatch strategies for intra-hour balancing, with focus on optimal predictive dispatch strategies and investigating alternate optimality criteria and product constraints. In Section II, context, benefits and methods for predictive dispatch are introduced. In Section III, a framework for formulation and performance evaluation of operation strategies with optimal predictive dispatch is presented. The framework is applied to a case study in Section IV for balancing wind power where the effects of variations in the parameters of alternate dispatch strategies are evaluated on relevant performance criteria.

\section{BACKGROUND AND METHODS}

\section{A. Balancing Power Products}

Balancing power here is considered as a subset of active power ancillary services activated to restore and maintain system and inter-area exchange balances. The technical definitions and the market conditions for control reserves differ between countries [12]. Aiming at a more unified definition of reserve types in Europe, ENTSO-E (European Network of TSOs for Electricity) recently introduced a policy framework [13] which identifies three main types of reserves: i) Frequency Containment Reserves (FCR) is a local automatic control used to counteract the frequency deviations and restore the balance between generation and demand, ii) Frequency Restoration Reserves (FRR) is a centralized automatic control used to bring the frequency back to its nominal value and restore the power flow of the tie-lines between neighboring systems to their target values and iii) Replacement Reserves (RR) refer to manual changes in the dispatch of generating units which are used to restore FCR and FRR and alleviate congestions. FCR and FRR are inherently reactive, i.e., activated automatically in response to measured deviations. In contrast, $\mathrm{RR}$ are activated manually with a more lenient response time (i.e. 15min), which enables their predictive dispatch. Replacement reserves are also procured through an hourly balancing market with gate closure time 45 minutes before actual operation [14].

\section{B. Predictive Dispatch of Regulating Power}

The predictive dispatch of regulating power can be based either on deterministic or on stochastic forecasts of the power imbalances during the real-time operation.

1) Deterministic Dispatch: The deterministic dispatch models employ point forecasts in order to predict the realization of the uncertain parameters such as wind power production. In general, the actual system imbalance $p_{t+k}^{i m b}$ at time $t+k$ corresponds to the realization of a random variable $\mathcal{P}_{t+k}^{i m b}$. A point forecast $\hat{p}_{t+k \mid t}^{i m b}$ is defined as $\hat{p}_{t+k \mid t}^{i m b}=\mathbb{E}\left[\mathcal{P}_{t+k \mid t}^{i m b}\right]$, denoting the expected imbalance at time $t+k$ conditional to the forecast issue time $t$. The deterministic dispatch model finds the optimal schedule $P^{M \text {,* }}$ of manual reserves that minimizes the objective function $J^{M}\left(P^{M}\right)$ as:

$$
\min _{P^{M}} J^{M}\left(P^{M}\right)
$$

s.t.

$$
\begin{aligned}
& h^{M}\left(P^{M}, \hat{P}^{i m b}\right)=0 \\
& g^{M}\left(P^{M}, \hat{P}^{i m b}\right) \leq 0
\end{aligned}
$$

where $\hat{P}^{i m b}$ is the vector of expected system imbalances. The equality constraints (2) include the system balance constraints and the decomposition of the energy supply curve into blocks. Constraint (3) specifies the operational limits of the power system as well as the dispatch limits of the regulating power bids. Given the vector $P^{i m b}$ of the actual imbalances, the automatic reserve dispatch $P^{A}$ is the vector that satisfies the real-time power balance and operational constraints:

$$
\begin{aligned}
& h^{A}\left(P^{A}, P^{i m b}, P^{M, *}\right)=0 \\
& g^{A}\left(P^{A}, P^{i m b}, P^{M, *}\right) \leq 0
\end{aligned}
$$

Constraint (4) requires that the remaining imbalance $P^{i m b}-$ $P^{M \text {,* }}$ will be covered by the automatic response, while constraint (5) ensures that this response respects the operational and reserve limits.

2) Stochastic Dispatch: Point forecasts provide a single value about the expected wind energy generation and they do not include any information about the associated uncertainty. The accurate representation of the uncertainty requires the consideration of the interdependence structure of the prediction errors. In the operational strategies, this stochasticity is represented using a set $S$ of scenarios spanning the full range of plausible realizations of the stochastic imbalance $\mathcal{P}_{t+k}^{i m b}$. Each scenario $s \in S$ is characterized by a vector of imbalances $P_{s}^{i m b}$, which respects the temporal interdependence structure of the prediction errors.

We model operational strategies as a two-stage stochastic programming problem, where the first stage (st1) decisions represent the activation of manual control reserves and the second stage (st2) represents the activation of automatic reserves at every given scenario. The stochastic dispatch model is described by the following optimization problem:

$$
\min _{P^{M}, P^{A}} J^{M, s t 1}\left(P^{M}\right)+\mathbb{E}_{s}\left[J_{s}^{M, s t 2}\left(P_{s}^{A}\right)\right]
$$

s.t.

$$
\begin{aligned}
& h_{s}\left(P^{M}, P_{s}^{A}, P_{s}^{i m b}\right)=0 \quad \forall s \\
& g_{s}\left(P^{M}, P_{s}^{A}, P_{s}^{i m b}\right) \leq 0 \quad \forall s
\end{aligned}
$$

where $\mathbb{E}_{s}[\cdot]$ is the expectation operator over the scenario set $S$. Constraints (7) and (8) are equivalent to (2) and (3) for every scenario $s \in S$.

The main advantage of the stochastic solution is that it optimizes simultaneously the two reserve types and provides an endogenous solution for the optimal amount of each reserve by weighing the expected costs and benefits of every alternative solution. Thus, the requirements for balancing power are not based on exogenous rules but they are included implicitly in the operational strategy of the TSO. In theory, the stochastic 
optimization solution yields the maximum system efficiency as it minimizes the objective function in expectation.

\section{Modeling Wind Uncertainty}

1) Methodology for the Generation of Wind Power Scenarios: The prediction uncertainty between different forecast horizons is modeled using a set scenarios taking into account the temporal interdependence structure of forecast errors [15]. Being at time $t$, write $\hat{f}_{t+k \mid t}$ the probabilistic forecast of the density function of wind power production $p_{t+k}$ at time $t+k$ and $\hat{F}_{t+k \mid t}$ the corresponding cumulative distribution function. For a single look-ahead time $k$, the realization $Y_{k}^{(t)}$ of the random variable $Y_{k}$ is defined as:

$$
Y_{k}^{(t)}=\hat{F}_{t+k \mid t}\left(p_{t+k}\right) \quad \forall t
$$

and follows a uniform distribution in the unit interval $Y_{k} \sim$ $U[0,1]$. Then, a standard normal random variable $X_{k} \sim$ $N[0,1]$ is obtained using the following transformation:

$$
X_{k}^{(t)}=\Phi^{-1}\left(Y_{k}^{(t)}\right) \quad \forall t
$$

where $\Phi^{-1}$ is the inverse of the Gaussian cumulative distribution function. Applying this transformation into all the uniform variables $Y_{k}^{(t)}, k=1 \ldots K$, where $K$ the maximum prediction horizon, one can get a random vector $\boldsymbol{X}=$ $\left(X_{1}, X_{2}, \ldots, X_{k}\right)^{T} \sim N\left(\mu_{0}, \Sigma\right)$, with $\mu_{0}$ the vector of mean values being a zero vector and $\Sigma$ the covariance matrix presenting the temporal interdependence between the elements of $\boldsymbol{X}$.

In order to produce $S$ scenarios at time $t$ and for the whole forecast horizon, i.e., $k=1, \ldots, K$, a multivariate Normal random number generator with zero mean and covariance matrix $\Sigma$ is used to draw $S$ realizations of $\boldsymbol{X}$. Denote by $X^{(s)}$ the $s^{t h}$ of these $S$ realizations. Then, for each horizon $k$ the inverse probit function $\Phi$ is applied to each element $X^{(s)}$ of $\boldsymbol{X}$ in order to obtain $S$ realizations of $Y_{k}^{(s)}$ as:

$$
Y_{k}^{(s)}=\Phi\left(X_{k}^{(s)}\right) \quad \forall k, \forall s
$$

Finally, $S$ wind power production scenarios are generated applying the inverse cumulative distribution function $\hat{F}_{t+k \mid t}^{-1}$ to each component of $Y_{k}^{(s)}$ as:

$$
\hat{p}_{t+k \mid t}^{(s)}=\hat{F}_{t+k \mid t}^{-1}\left(Y_{k}^{(s)}\right) \quad \forall k, \forall s
$$

2) Wind Power Predictive Distributions: In real applications the predictive densities $\hat{f}_{t+k \mid t}$ can be obtained using nonparametric probabilistic forecasts, where no explicit assumption is made on the shape of the predictive densities. For the purpose of this paper, we employ Beta distributions $B(\alpha, \beta)$ for modeling the predictive densities $\hat{f}_{t+k \mid t}$, as suggested in [16]. The shape parameters $\alpha_{t+k \mid t}, \beta_{t+k \mid t}$ of the Beta distribution $B_{t+k \mid t}$, for each time $t$ and look-ahead time $k$ are calculated as:

$$
\begin{array}{r}
\alpha=\mu_{B}\left(\frac{\mu_{B}\left(1-\mu_{B}\right)}{\sigma_{B}^{2}}-1\right) \\
\beta=\left(1-\mu_{B}\right)\left(\frac{\mu_{B}\left(1-\mu_{B}\right)}{\sigma_{B}^{2}}-1\right)
\end{array}
$$

where $\mu_{B}$ and $\sigma_{B}^{2}$ denote the mean and the variance of $B(\alpha, \beta)$ and are different than the parameters $\mu_{0}, \Sigma$ of the Gaussian distribution used in (10). Note that in (13) and (14) we have omitted $t+k \mid t$ for brevity of notation.

The values of $\mu_{B, t+k \mid t}$ and $\sigma_{B, t+k \mid t}^{2}$ can be estimated from the available dataset of wind power measurements applying the Nadaraya-Watson kernel regression model:

$$
\begin{array}{r}
\mu_{B, t+k \mid t}=\frac{\sum_{k^{\prime}=1}^{K} W_{b_{k}}\left(t+k, t+k^{\prime}\right) p_{t+k^{\prime}}}{\sum_{k^{\prime}=1}^{K} W_{b_{k}}\left(t+k, t+k^{\prime}\right)} \\
\sigma_{B, t+k \mid t}^{2}=\frac{\sum_{k^{\prime}=1}^{K} W_{b_{k}}\left(t+k, t+k^{\prime}\right)\left(p_{t+k^{\prime}}-\mu_{t+k \mid t}\right)^{2}}{\sum_{k^{\prime}=1}^{K} W_{b_{k}}\left(t+k, t+k^{\prime}\right)}
\end{array}
$$

which is a nonparametric approach to estimate the conditional expectation of a random variable. The $W_{b_{k}}$ denotes the Gaussian kernel with smoothing parameter $b_{k}$ which controls the bandwidth of the kernel function. In order to simulate in a simple manner the known characteristics of forecast uncertainty, where the potential magnitude of forecast errors increases with the lead time, the value of $b_{k}$ is proportional to the look-ahead time $k$ :

$$
b_{k}=b_{0}+b_{1} \sqrt{k}
$$

The covariance matrix $\Sigma$ is a symmetric $k \times k$ matrix with all its diagonal elements equal to 1 . The off-diagonal elements of $\Sigma$ follow the structure:

$$
\Sigma=\left(\begin{array}{cccc}
1 & e^{-1 / \eta} & \ldots & e^{-(k-1) / \eta} \\
e^{-1 / \eta} & 1 & \ldots & e^{-(k-2) / \eta} \\
\vdots & \vdots & \ddots & \vdots \\
e^{-(k-1) / \eta} & e^{-(k-2) / \eta} & \ldots & 1
\end{array}\right)
$$

and $\eta$ is set equal to 7. For further discussion on the appropriate range parameter of $\eta$ in such a covariance model, the reader may refer to [17]. Note, that this formulation implies that $\Sigma$ is independent of time $t$. In practical applications the covariance matrix can be adaptively estimated using a recursive estimation method.

\section{DEFINITION AND EVALUATION OF OPERATIONAL STRATEGIES}

This section provides the definition of the operational strategies and proposes several criteria in order to assess their performance.

\section{A. Definition of Operational Strategies}

The definition of operational strategies is essential because they provide the necessary link between the available balancing power products and the decision-making policy of the TSO, as illustrated in Fig. 1. The main components of an operational strategy are: 


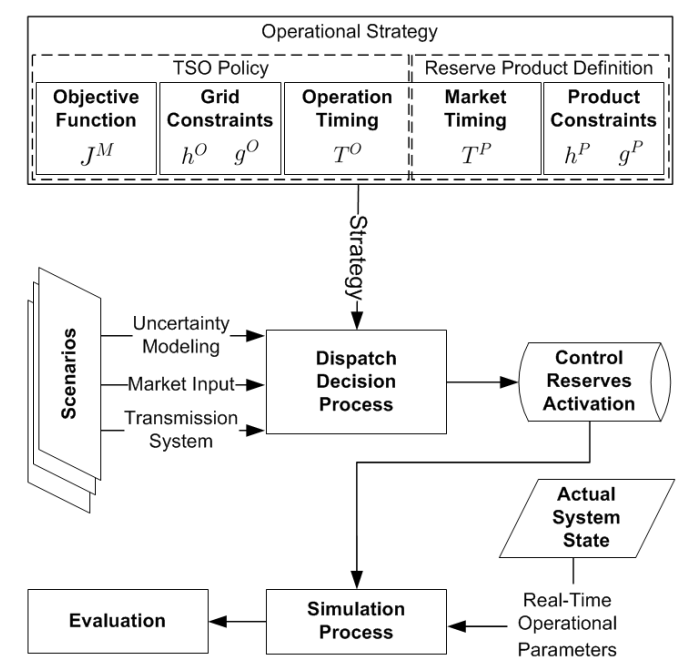

Fig. 1: Illustration of operational strategies concept for control reserves activation.

1) The TSO Policy.

a) The objective function $J^{M}$.

b) The grid constraints $h^{O}$ and $g^{O}$.

c) The operation timing $T^{O}$.

2) The reserve product definition.

a) Market timing $T^{P}$.

b) Product constraints $h^{P}$ and $g^{P}$.

The objective function $J^{M}$ reflects the interests of the TSO during the balancing operation. The grid constraints $h^{O}$ and $g^{O}$ represent the technical limitations of the power network, e.g., transfer capacities and voltage limits, that should be respected during the operation. The operation timing $T^{O}$ refers to the scheduling horizon and the lead time $T^{l t}$, i.e., the time interval between decision-making and actual operation. The term market timing $T^{P}$ includes the temporal parameters of the balancing market such as the gate closure time and the interval between two consecutive clearings. Finally, product constraints $h^{P}$ and $g^{P}$ are related to the bid structure of the regulating market.

\section{B. Simulation and Evaluation}

The actual operation of the power system in different test cases is simulated when uncertainty is revealed, i.e., the realized imbalances and the real-time parameters of the power system are known, e.g., grid constraints, actual RES production and fluctuations. Considering the specific characteristics of each power system, the performance of a balancing strategy depends upon: i) the penetration level of non-dispatchable generation, ii) the interconnections with the neighboring systems, iii) the composition of the controllable generation portfolio and iv) the existing market scheme. The effectiveness of the operational strategies is assessed with respect to different criteria such as: i) the total operating cost, ii) the energy utilization and iii) the maximum power capacity of manual and automatic reserves.

\section{CASE STUDY}

This section illustrates the formulation of different operational strategies and assesses their performance based on the above criteria using a small-scale case study.

\section{A. Problem Statement}

Here, we investigate specific variations on the current practice for reserve dispatch [14] and assess the effectiveness of the corresponding operational strategies. The following case study considers alternative objective functions, lead times and minimum up time of the regulating bids.

\section{B. Data}

A set of publicly available wind power measurements provided by the Australian Energy Market Operator [18] were used to generate wind power scenarios following the methodology described in Section II. The full data set contains wind power measurements, with 5-minute resolution, for 23 onshore wind farms located in Western Australia. For the purpose of the case study, we have selected three wind farms spread over an area approximately equal to Denmark. A total installed wind power capacity of $200 \mathrm{MW}$ is assumed to be equally dispersed among the three wind farms.

Figure 2 provides an example of 50 randomly picked scenarios, produced using the methodology presented in Section II-C, and the corresponding wind power measurements normalized by the installed capacity of the corresponding wind farm. The lead time is 15 minutes and the prediction horizon is three hours. The increasing range of scenarios for higher look-ahead times reflects the uncertainty of the wind power predictions.

Data of the bids for up and down regulation are given in Table I. Each bid is defined as a combination of offered energy quantity (block width) either for up $p_{t}^{u p}$ or down $p_{t}^{d n}$ regulation with a bid price of $\lambda_{t}^{u p}$ and $\lambda_{t}^{d n}$, respectively. The activation price for automatic up $\left(c^{a, u p}\right)$ and down $\left(c^{a, d n}\right)$ reserves is equal to $100 € / \mathrm{MWh}$ and $5 € / \mathrm{MWh}$ respectively. These offers refer only to energy prices, assuming availability of sufficient reserve capacity. The last two columns of Table I contain the deviation of the balancing prices from the day-ahead price $\lambda_{t}^{s}=30 € / \mathrm{MWh}$, i.e., $\Delta \lambda_{t}^{u p / d n}=\lambda_{t}^{u p / d n}-\lambda_{t}^{s}$. The corresponding values for the automatic reserves are $\Delta c^{a, u p}=70$ $€ / \mathrm{MWh}$ and $\Delta c^{a, d n}=-25 € / \mathrm{MWh}$. Note that $\Delta \lambda_{t}^{d n}$ and $\Delta c^{a, d n}$ are negative, since the generators are willing to pay the TSO to reduce their output as they save their fuel costs. For the purpose of this case study, we assume that all regulating offers and the day-ahead price are the same throughout the considered period of one week. The time discretization is 5 minutes and the scheduling horizon is one hour.

\section{Case Study Assumptions}

The operational strategies proposed here are based on the following assumptions:

1) Only wind power uncertainty is considered and it is assumed that demand and "firm" resource availability can be perfectly forecast. Hence, the balancing operation has to compensate only for wind power deviations from the 

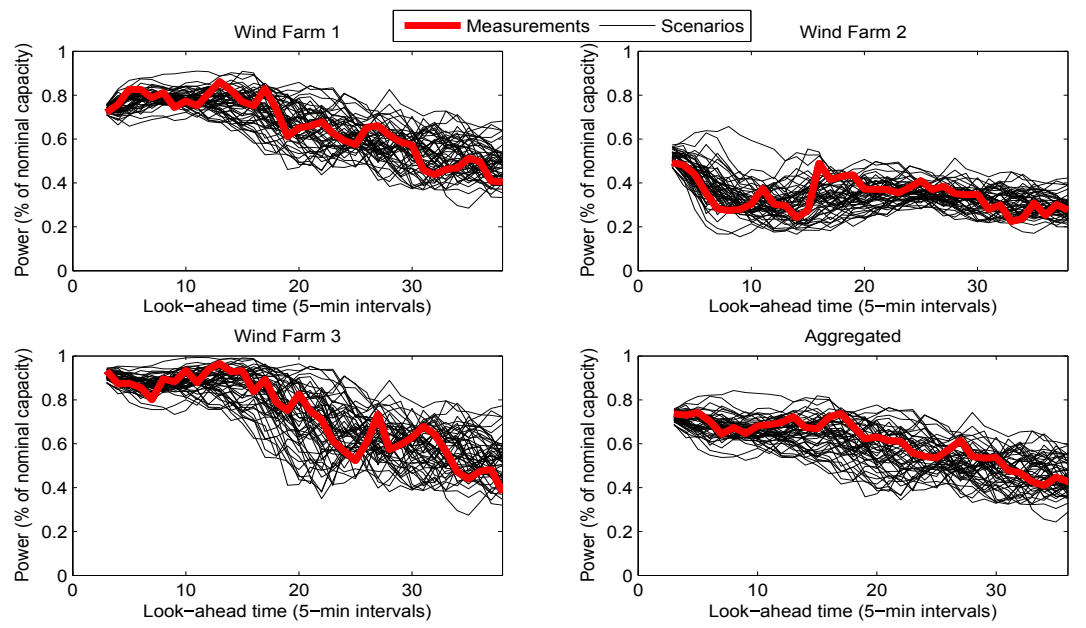

Fig. 2: Illustration of 50 alternative wind power scenarios for prediction horizon of 3 hours with lead time 15 min and the corresponding wind power measurements. X-axis index: number of 5-minute intervals.

TABLE I: REGULATING POWER BIDS

\begin{tabular}{|c|c|c|c|c|c|c|}
\hline \multirow{2}{*}{ Block \# } & \multicolumn{2}{|c|}{ Up Regulation } & \multicolumn{2}{c|}{ Down Regulation } & \multicolumn{2}{c|}{ Deviation from $\lambda_{t}^{s}$} \\
\cline { 2 - 7 } & $\begin{array}{c}p_{t}^{u p} \\
(\mathrm{MWh})\end{array}$ & $\begin{array}{c}\lambda_{t}^{u p} \\
(€ / \mathrm{MWh})\end{array}$ & $\begin{array}{c}p_{t}^{d n} \\
(\mathrm{MWh})\end{array}$ & $\begin{array}{c}\lambda_{t}^{d n} \\
(€ / \mathrm{MWh})\end{array}$ & $\begin{array}{c}\Delta \lambda_{t}^{u p} \\
(€ / \mathrm{MWh})\end{array}$ & $\begin{array}{c}\Delta \lambda_{t}^{d n} \\
(€ / \mathrm{MWh})\end{array}$ \\
\hline \hline 1 & 10 & 40 & 10 & 10 & 10 & -20 \\
\hline 2 & 15 & 45 & 20 & 15 & 15 & -15 \\
\hline 3 & 20 & 60 & 15 & 20 & 30 & -10 \\
\hline 4 & 30 & 70 & 35 & 25 & 40 & -5 \\
\hline
\end{tabular}

day-ahead dispatch. The day-ahead market is cleared using the expected hourly wind power production. Any other source of uncertainty, e.g., load deviations or equipment failures (generation unit or transmission line outage), can be represented similarly using appropriate scenarios.

2) The cost of balancing power emanates only from energy production. The TSO has full information about the cost of automatic reserves and the supply curves for manual regulation during the balancing operation. The supply curves are the aggregation of the energy bids (blocks of quantity-price offers) submitted by the market players.

3) The optimal dispatch of balancing power neglects any capacity reserve markets. This is a reasonable assumption considering that capacity markets are cleared in advance of the actual operation of the power system [19].

4) The power system follows the zonal pricing scheme and thus the local network constraints are not considered. This is in compliance with the market structure of Nordpool.

5) During the decision-making for balancing operation, the TSO has full information about the wind uncertainty from all the wind farms of the system in the form of scenarios.

6) The evaluation of automatic reserves is performed using the same time discretization as the dispatch optimization.

\section{Mathematical Formulation}

The stochastic dispatch problem for the activation of control reserves is described by the following minimization problems.
Objective $J_{I}^{M}$ : Cost minimization

$$
\begin{aligned}
\min _{P^{M}, P^{A}} J_{I}^{M}= & \sum_{t=1}^{T}\left(\sum_{m=1}^{M} \lambda_{t}^{u p}(m) p_{t}^{u p}(m)-\sum_{n=1}^{N} \lambda_{t}^{d n}(n) p_{t}^{d n}(n)\right)+ \\
& \sum_{t=1}^{T} \sum_{s=1}^{S} \pi_{s}\left(c^{a, u p} P_{t, s}^{a, u p}-c^{a, d n} P_{t, s}^{a, d n}\right)
\end{aligned}
$$

Objective $J_{I I}^{M}$ : Minimum automatic reserves utilization

$$
\min _{P^{M}, P^{A}} J_{I I}^{M}=\sum_{t=1}^{T} \sum_{s=1}^{S} \pi_{s}\left(P_{t, s}^{a, u p}+P_{t, s}^{a, d n}\right) d t
$$

s.t.

Grid constraint (power balance) $h^{O}$ :

$$
P_{t}^{u p}-P_{t}^{d n}+P_{t, s}^{a, u p}-P_{t, s}^{a, d n}=\Delta P_{t, s}^{w} \quad \forall t, \forall s
$$

Reserve products $h^{P}, g^{P}$ :

$$
\begin{gathered}
P_{t}^{u p}=\sum_{m=1}^{M} p_{t}^{u p}(m) \quad \forall t \\
P_{t}^{d n}=\sum_{n=1}^{N} p_{t}^{d n}(n) \quad \forall t \\
0 \leq p_{t}^{u p}(m) \leq p_{t}^{u p, \max }(m) \quad \forall t, \forall m \\
0 \leq p_{t}^{d n}(n) \leq p_{t}^{d n, \max }(n) \quad \forall t, \forall n \\
P_{t}^{u p}=P_{\tau}^{u p} \quad t \geq \tau \text { and } t \leq \tau+T_{\text {min }}^{u p}-1 \\
P_{t}^{d n}=P_{\tau}^{d n} \quad t \geq \tau \text { and } t \leq \tau+T_{\text {min }}^{u p}-1
\end{gathered}
$$$$
0 \leq p_{t}^{u p}(m) \leq p_{t}^{u p, \max }(m) \quad \forall t, \forall m
$$$$
0 \leq p_{t}^{d n}(n) \leq p_{t}^{d n, \max }(n) \quad \forall t, \forall n
$$$$
P_{t}^{u p}=P_{\tau}^{u p} \quad t \geq \tau \text { and } t \leq \tau+T_{\text {min }}^{u p}-1
$$

The objective function (19) to be minimized is the expected cost of balancing operation. This cost arises from two terms:

1) the deployment of manual up and down reserve blocks, $p_{t}^{u p}(m)$ and $p_{t}^{d n}(n)$ respectively, with corresponding marginal cost of energy equal to $\lambda_{t}^{u p}(m)$ and $\lambda_{t}^{d n}(n)$, where $M$ and $N$ indicate the number of up and down bids. 
TABLE II: OPERATIONAL STRATEGIES

\begin{tabular}{|l|c|c|c|c|c|c|}
\cline { 2 - 7 } \multicolumn{1}{c|}{} & $\mathrm{S} 1$ & $\mathrm{~S} 2$ & $\mathrm{~S} 3$ & $\mathrm{~S} 4$ & $\mathrm{~S} 5$ & $\mathrm{~S} 6$ \\
\hline$T_{\min }^{u p}(\mathrm{~min})$ & 60 & 60 & 60 & 60 & 30 & 30 \\
\hline$T^{l t}(\mathrm{~min})$ & 15 & 30 & 15 & 30 & 15 & 15 \\
\hline Objective & $J_{I}^{M}$ & $J_{I}^{M}$ & $J_{I I}^{M}$ & $J_{I I}^{M}$ & $J_{I}^{M}$ & $J_{I I}^{M}$ \\
\hline
\end{tabular}

2) the deployment of automatic up and down reserves, $P_{t}^{a, u p}$ and $P_{t}^{a, d n}$ respectively, with activation price equal to $c^{a, u p}$ and $c^{a, d n}$.

Note that the positive down-regulation bids $\lambda_{t}^{d n}(n)$ and $c^{a, d n}$ enter the objective function (19) with a negative sign, since they represent a repurchase price.

An alternative objective function (20) to be minimized is the expected utilization of automatic reserves (in terms of energy). For each time period $t$ of the scheduling horizon, the energy production of automatic reserves is given as the product of the deployed automatic reserves (up and down) with the duration $d_{t}$ of each time period $t$. In both objective functions $\pi_{s}$ denotes the probability of occurrence for scenario $s$.

In the above two-stage problem formulation, the decisions that are taken in the first stage, i.e., deployment of manual up and down reserves, cannot be modified once the uncertainty in the system is revealed. In turn, the second stage variables represent the actual operation of the power system where decisions can be adapted according to the realized scenario.

The power balance constraint (21) requires that the wind deviations $\Delta P_{t, s}^{w}$ at every scenario $s$ are covered by the dispatched manual and automatic reserves (equivalent to a market clearing constraint). Constraints (22) and (23) represent the block energy offers submitted to the balancing market from the generators who provide up and down regulation respectively. In addition, constraints (24) and (25) specify the upper and the lower limits of each block offer. Finally, constraints (26) and (27) guarantee that the re-dispatch of manual reserves can be performed according to the time-frame of the existing balancing market. In this context, $\tau$ represents the discrete time-steps when re-dispatching is allowed while $T_{\text {min }}^{u p}$ is the time resolution of the balancing market, i.e., the number of time steps between two successive trading periods.

\section{E. Parameters of Operational Strategies}

Different operational strategies are formulated in order to investigate the effect of their components on the performance of the power system. Table II summarizes the main parameters for the six operational strategies ( $\mathrm{S} 1$ to S6) considered in this case study. Reducing the minimum up time $T_{\text {min }}^{u p}$, from 60 to 30 minutes, allows more frequent re-dispatching of the balancing resources according to the predicted imbalances. In addition, lower lead time, from 30 to 15 minutes, is chosen in order to account for the improved quality the available forecasts of wind production. Objective function $J_{I}^{M}$ refers to cost minimization aiming at the economic efficiency of balancing operation. Finally, using objective $J_{I I}^{M}$, the TSO is able to reduce directly the needs for automatic reserves and increase their availability during a contingency event.
TABLE III: PERFORMANCE RESULTS

\begin{tabular}{|lc|c|c|c|c|c|c|}
\hline Performance Metric & S1 & S2 & S3 & S4 & S5 & S6 \\
\hline \hline \multicolumn{2}{|l|}{ Total Cost $\left(\times 10^{3} €\right)$} & 652.83 & 662.29 & 907.01 & 909.51 & $\mathbf{6 0 2 . 4 9}$ & 810.58 \\
\hline Max $P_{t}^{u p}(\mathrm{MW})$ & $\mathbf{4 6 . 2 7}$ & 46.36 & 48.09 & 47.97 & 46.95 & 53.73 \\
\hline Max $P_{t}^{d n}(\mathrm{MW})$ & $\mathbf{5 2 . 6 9}$ & 54.29 & 76.25 & 79.51 & 66.39 & 80 \\
\hline Max $P_{t}^{a, u p}(\mathrm{MW})$ & 35.94 & 37.26 & 65.20 & 73.13 & $\mathbf{3 0 . 3 0}$ & 39.25 \\
\hline Max $P_{t}^{a, d n}(\mathrm{MW})$ & 104.76 & 102.37 & 100.73 & $\mathbf{9 6 . 6 9}$ & 105.97 & 103.93 \\
\hline$E^{M}$ & $(\mathrm{GWh})$ & $\mathbf{2 . 1 7}$ & 2.21 & 2.53 & 2.56 & 2.23 & 2.54 \\
\hline$E^{A}$ & $(\mathrm{GWh})$ & 1.25 & 1.23 & 1.06 & 1.05 & 1.04 & $\mathbf{0 . 8 6}$ \\
\hline
\end{tabular}

\section{F. Simulation Results and Evaluation of Operational Strate-} gies

Figure 3 provides an illustration of the actual dispatch of automatic and manual reserves for the different operational strategies. It can be observed that reserve activation follows a similar pattern in all strategies, which is mainly dictated by the sign and the magnitude of the forecasted imbalance. However, the exact amount of each reserve type activated in every period changes depending on the applied operational strategy. Strategies S3, S4 and S6 activate higher amounts of manual reserves in to order to decrease the utilization of automatic reserves. On the contrary, strategies S1, S2 and S5 find the optimal combination of available balancing resources in order to minimize the expected cost of the system. It should be noted, that during certain periods it is possible to have counteractivation of manual and automatic reserves with different sign, i.e., up and down regulation. This can be justified by the fact that the actual imbalance has opposite direction than the one predicted in the scenarios.

Table III provides the values of some relevant performance metrics for each operating strategy. It can be observed that the total balancing cost reduces significantly using operational strategies that employ objective function $J_{I}^{M}$ compared to those that use objective function $J_{I I}^{M}$. On the contrary, using objective function $J_{I I}^{M}$, the reduction of automatic reserve utilization $E^{A}$, despite their higher activation cost, is not able to compensate the cost increase entailed by the larger volumes of manual reserves utilization $E^{M}$. Shorter lead time has a marginal effect on the balancing operation, reducing the needs for manual reserves both in terms of energy and capacity but increasing to a small extent the needs for automatic resources. Finally, the decrease in minimum up time has the most profound positive effect on the balancing operation, regarding both the cost and the reserve needs. This underlines the importance of market rules and indicates that increased flexibility of the resources may bring considerable benefits in the power system.

\section{CONCLUSIONS AND FUTURE WORK}

This paper provides a framework for the definition and formulation of operational strategies for reserve activation, which can be used to evaluate the impact of relevant parameters in the dispatch results. Our study focuses on the predictive dispatch of regulating power needed to cope with wind uncertainty. Alternative operational strategies in terms 

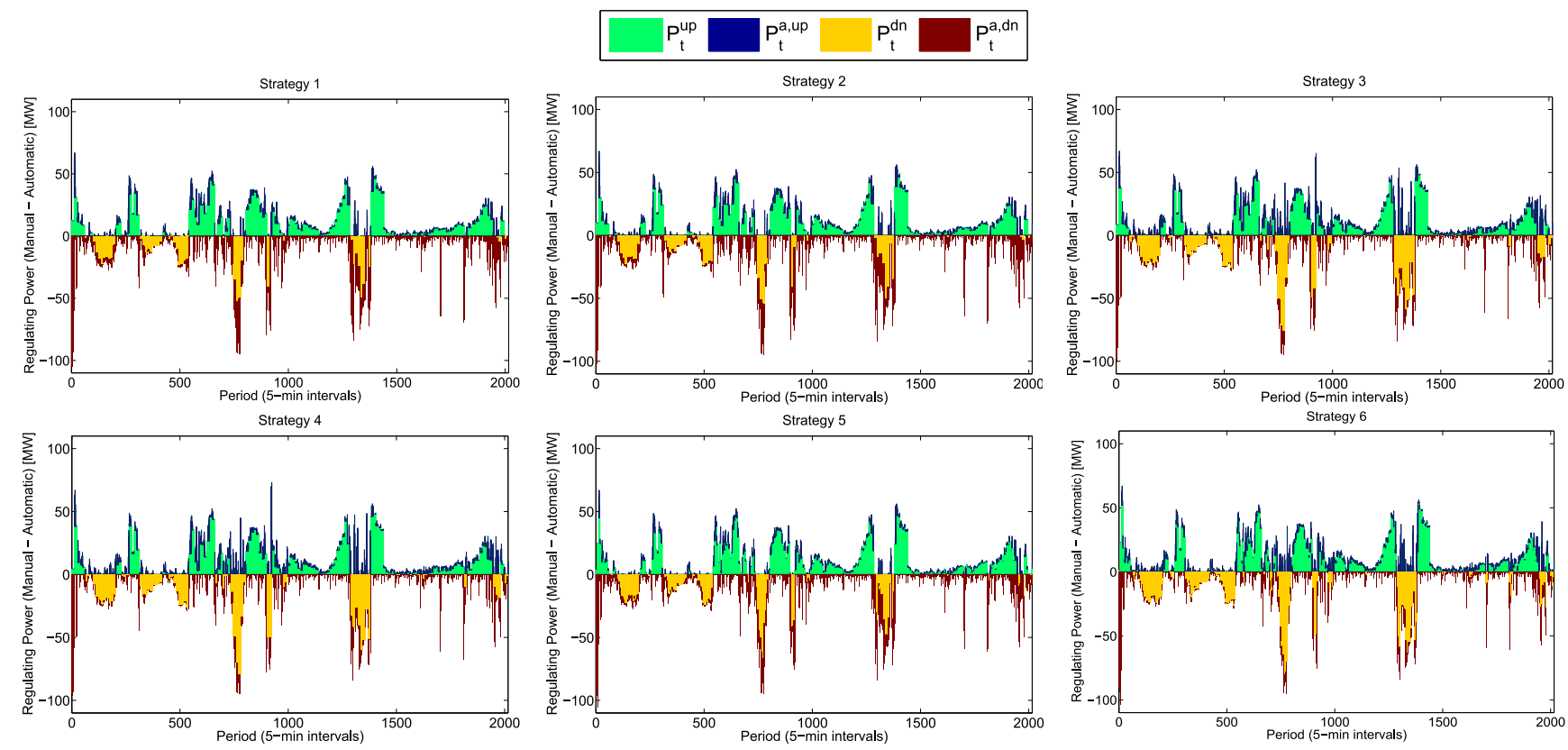

Fig. 3: Dispatch of manual and automatic reserves for the different operational strategies. X-axis index: number of 5-minute intervals.

of product specification, timing and availability of balancing resources are formulated and evaluated using performance criteria. The results of the case study have shown that variations in performance indices can be significant and these trade-offs, i.e., expected cost of balancing operation and expected utilization of automatic reserves, should be considered during the decision-making for balancing operation. The most significant impact on the performance metrics arises from variations in the balancing products definition, which highlights also the importance of power system flexibility.

Future work will investigate more variations of the balancing power products, including energy and ramping limits, as well as the representation of network constraints. In addition, different time resolution between the dispatch optimization and the simulation of actual operation will be also considered. Finally, the proposed methodology will be tested using real data in order to confirm the validity of the results and investigate possible improvements.

\section{REFERENCES}

[1] M. MacDonald, "Impact Assessment on European Electricity Balancing Market," European Commission, Tech. Rep., 2013. [Online]. Available: http://ec.europa.eu/energy/gas_electricity/studies/ doc/electricity/20130610_eu_balancing_master.pdf

[2] A. Papavasiliou, S. S. Oren, and R. P. O'Neill, "Reserve requirements for wind power integration: A scenario-based stochastic programming framework," Power Systems, IEEE Transactions on, vol. 26, no. 4, pp. 2197-2206, 2011.

[3] J. M. Morales, A. J. Conejo, and J. Pérez-Ruiz, "Economic valuation of reserves in power systems with high penetration of wind power," Power Systems, IEEE Transactions on, vol. 24, no. 2, pp. 900-910, 2009.

[4] A. Tuohy, P. Meibom, E. Denny, and M. O'Malley, "Unit commitment for systems with significant wind penetration," Power Systems, IEEE Transactions on, vol. 24, no. 2, pp. 592-601, 2009.

[5] M. Zugno and A. J. Conejo, "A robust optimization approach to energy and reserve dispatch in electricity markets," Technical University of Denmark, Tech. Rep., 2013.
[6] M. A. Matos and R. J. Bessa, "Setting the operating reserve using probabilistic wind power forecasts," Power Systems, IEEE Transactions on, vol. 26, no. 2, pp. 594-603, 2011.

[7] A. Hansen, A. Orths, K. Falk, and N. K. Detlefsen, "Danish fossil independent energy system 2050 - from strategic investigations to intrahour simulation of balancing issues," in In Proc. 10th Internation Workshop on Large-Scale Integration of Wind Power into Power Systems, Aarhus, Denmark, October 2011, 2011.

[8] S. Stoft, Power system economics: Designing markets for electricity. Wiley-IEEE Press, 2002.

[9] B. Wang and B. Hobbs, "Flexiramp market design for real-time operations: Can it approach the stochastic optimization ideal?" in In Proc. Power and Energy Society General Meeting (PES), 2013 IEEE. IEEE, 2013.

[10] L. Xu and D. Threteway, "Flexible ramping products-second revised draft final proposal," California ISO (CAISO), Tech. Rep., 2013.

[11] PJM [Online]. Available: www.pjm.com.

[12] Y. G. Rebours, D. S. Kirschen, M. Trotignon, and S. Rossignol, "A survey of frequency and voltage control ancillary servicesPart i: Technical features," Power Systems, IEEE Transactions on, vol. 22, no. 1, pp. 350-357, 2007.

[13] ENTSO-E, Network Code on Load-Frequency Control and Reserves, ENTSO-E Std., 2013. [Online]. Available: https://www.entsoe.eu/fileadmin/user_upload/_library/resources/ LCFR/130628-NC_LFCR-Issue1.pdf

[14] D. Morales Bondy, K. Heussen, G. Tarnowski, and L. Hansen, "Operational Scenario: Manual regulating power," iPower Project - WP4, Tech. Rep., 2013.

[15] P. Pinson, H. Madsen, H. A. Nielsen, G. Papaefthymiou, and B. Klöck1, "From probabilistic forecasts to statistical scenarios of short-term wind power production," Wind energy, vol. 12, no. 1, pp. 51-62, 2009.

[16] A. Fabbri, T. Gomez San Roman, J. Rivier Abbad, and V. Mendez Quezada, "Assessment of the cost associated with wind generation prediction errors in a liberalized electricity market," Power Systems, IEEE Transactions on, vol. 20, no. 3, pp. 1440-1446, 2005.

[17] P. Pinson and R. Girard, "Evaluating the quality of scenarios of shortterm wind power generation," Applied Energy, vol. 96, pp. 12-20, 2012.

[18] AEMO [Online]. Available: www.aemo.com.au.

[19] Energinet.dk, "Energinet.dk's ancillary services strategy," 2011. 